\title{
Frequently asked questions about generic medicines
}

\author{
Andrew J McLachlan, Professor of Pharmacy (Aged Care), Centre for Education and \\ Research on Ageing, Concord Repatriation General Hospital and Faculty of Pharmacy, \\ University of Sydney; lqbal Ramzan, Professor of Pharmaceutics, Faculty of Pharmacy, \\ University of Sydney; and Robert W Milne, Associate Professor, Sansom Institute, \\ School of Pharmacy and Medical Sciences, University of South Australia, Adelaide
}

\section{Summary}

In Australia, generic products must be bioequivalent to the innovator brand name product, or the market leader, before they are approved. Australia has rigorous scientificallybased evaluation procedures for generic medicines based on the internationally accepted principle of bioequivalence. Under the Pharmaceutical Benefits Scheme, generic substitution is only permitted if two products are bioequivalent. Consumers should be encouraged to know and record the name of the active ingredient in the medicines they are receiving to avoid confusion between different brands of medicines. Healthcare professionals have a key role in helping consumers understand any real or perceived differences (or lack thereof) between different brands of medicines. Prescribing generics helps to contain health costs.

Key words: bioequivalence, pharmacokinetics.

(Aust Prescr 2007;30:41-3)

\section{Introduction}

When the patent of an innovator drug expires, other manufacturers can make generic versions. A generic drug contains the same active ingredient as another product, but is marketed under a different name. In Australia, the Pharmaceutical Benefits Advisory Committee (PBAC) recognises the interchangeability of different brands containing the same active ingredient, providing these brands are proven to be bioequivalent. ${ }^{1,2,3,4}$

\section{What is bioequivalence?}

Two products are bioequivalent when they produce such similar plasma concentrations of the active ingredient that their clinical effects can be expected to be the same.

In a standard bioequivalence test both products are administered on separate occasions to healthy volunteers.
Bioequivalence is then determined by comparing the peak plasma concentration $\left(\mathrm{C}_{\max }\right)$, time to achieve a maximal concentration ( $T_{\text {max }}$ ) and the extent of absorption (area under the concentration-time curve, AUC) of the products (Fig. 1).

These studies are well suited to identifying potentially significant differences in the delivery characteristics of the active substance of different products. The same bioequivalence principles apply to new drugs when different formulations of an active ingredient are compared.

Bioequivalent products are marked with a superscript $a$ or $b$ in the Schedule of Pharmaceutical Benefits. ${ }^{5}$

\section{Is bioequivalence clinically important?}

Yes, only those products that have been proven to be bioequivalent should be used interchangeably. On scientific grounds there is no reason to be concerned about substituting a generic product for a branded product that is flagged as being bioequivalent. ${ }^{5}$

\section{Fig. 1}

\section{Bioequivalence analysis - a hypothetical bioequivalence study}

Mean concentration-time curves for two brands of a drug after single oral doses

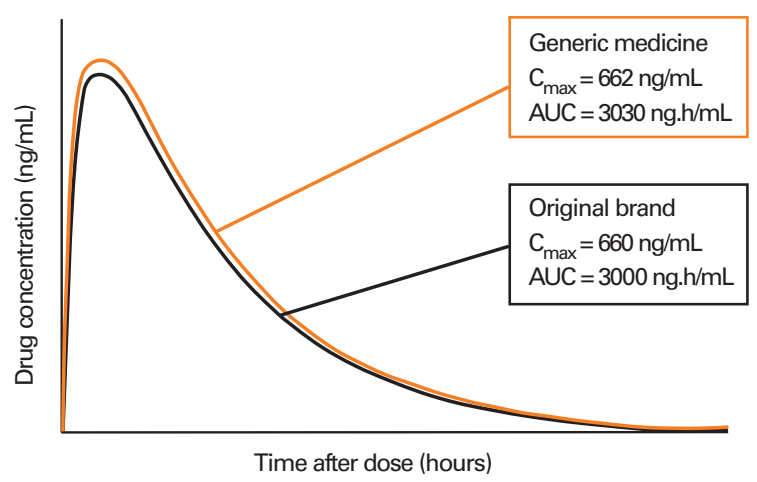

The original brand:generic medicine ratio for AUC is 0.99 (90\% $\mathrm{Cl} 0.91$ to 1.04 ) and for $\mathrm{C}_{\max }$ is 0.99 (90\% $\mathrm{Cl} 0.92$ to 1.07 ).

$\mathrm{C}_{\max }$ peak plasma concentration

AUC area under the concentration-time curve

$\mathrm{Cl}$ confidence interval

Reprinted with permission from NPS News 2006;44:3. 
Switching inequivalent products may lead to lower or higher blood concentrations of a drug in a patient. This may increase the risk of therapeutic failure or drug-related toxicity.

The precise extent to which inequivalence between two formulations will affect the clinical response depends on their pharmacological and/or therapeutic properties. It depends specifically on which part of the drug concentration-effect curve is affected by any concentration difference. ${ }^{4}$ For example, if the drug is usually dosed close to the upper flat part of the doseresponse curve, then large changes in plasma concentration will result in only small changes in therapeutic response or adverse effects. Theoretically, this is a greater concern for drugs with a narrow therapeutic index, such as carbamazepine, digoxin and sodium valproate. However, this is not as problematic as may be predicted because patients taking these drugs are generally closely monitored (either by measuring concentrations or effects). For drugs with wider safety margins, there should be no concerns about a change in response when switching from one bioequivalent brand to another.

\section{Which medicines should not be substituted?}

Products that are not bioequivalent should not be substituted for each other. For example, metoprolol is available as both an intermediate release and a modified release tablet. These dose forms are not bioequivalent and should not be substituted.

There are two innovator brands of warfarin available in Australia. These have not been proven to be bioequivalent and so it is recommended that warfarin products should not be substituted.

There has been considerable debate regarding the bioequivalence of drugs with a narrow therapeutic index, that is, drugs for which a small change in blood drug concentration leads to significant change in therapeutic response or toxicity. ${ }^{6}$ These drugs generally display relatively minor variability within a patient from day to day but often display considerable variability between patients. ${ }^{4,6}$ Taken together this implies that the dose required to achieve the same concentration in the body, and therefore the same pharmacological effect, might be quite different between different patients. However, within a patient the dose requirements are unlikely to vary greatly over time and between doses while the patient is clinically stable. Bioequivalence principles and criteria equally apply to medicines with a narrow safety margin. ${ }^{6,7}$

\section{Can people have a reaction to the excipients in different products?}

Yes, although adverse reactions to excipients are rare.

Pharmaceutical products contain the active pharmacological ingredient and a range of excipients that are designed to deliver the active drug optimally in a reliable and reproducible manner. These excipients can be diluents, binders, fillers, surfactants, lubricants, coatings and dyes. Excipients are generally considered 'inactive', but there is some evidence to suggest that excipients can have an impact on patient tolerability. ${ }^{8}$ The main risk is allergy or intolerance to a specific ingredient such as lactose. The range of excipients used pharmaceutically is small, and the type used in individual products must be carefully chosen so that bioequivalence is achieved. The quality and safety of all excipients are carefully reviewed by the Therapeutic Goods Administration (TGA) and excipients can only be used if they are safe and non-toxic. It may not be possible to determine which ingredients in either generic or branded products may cause an allergic reaction given that formulations are likely to be similar. Patients who are aware of their allergies can refer to the ingredients listed in the Consumer Medicines Information that accompanies the product.

\section{How can patients avoid being confused by the brand name of generic products?}

Patients should be encouraged to know and record the name of the active ingredient in the medicine they are taking rather than the product brand name. In this way a patient will understand that the same medicine may be available in different brands. This has implications for the way medicines are labelled. Ideally, the active ingredient in the product should be displayed with greater or equal prominence to the brand name on the packaging as recommended by the TGA in the 'Best practice guideline on prescription medicine labelling'. ${ }^{9}$

Public hospitals are likely to only have one or two brands of a medicine and these are often generic products. As patients move in and out of hospital it is likely that generic substitution will occur to a greater extent. This reinforces the need for patients to be aware of and carry a list of the name of the active ingredient or generic name of their medicines to maintain effective management of their condition. ${ }^{10}$

When deciding whether to substitute a generic product for a branded product, one must always consider the patient's understanding of their medicines and the risk of medication misadventure. Discuss this with the patient and provide appropriate information. ${ }^{3}$

If there is potential for confusion on the part of the patient and there is a risk of dose duplication, then generic substitution may need to be avoided (independent of the drug involved) unless the patient or carer fully understands the difference between the various brands of the same medicine. Clearly elderly patients, those with cognitive impairment and patients taking multiple medicines for serious chronic illness are at greatest risk of misadventure from their drugs.

\section{Do community pharmacists make a bigger profit if they substitute a generic drug?}

Not necessarily. Under the Brand Premium Policy of the Pharmaceutical Benefits Scheme (PBS), pharmacists are allowed to substitute a generic product when a branded product is prescribed, unless the prescriber directs otherwise.

The PBS provides a subsidy up to the price of the cheapest brand of a drug in a particular therapeutic area. This often creates 
a price difference between generic and branded products.

The pharmacist's profit margin varies from drug to drug and product to product. In the past, cost savings for community pharmacists arose when they purchased bulk orders of generic drugs directly from manufacturers. This issue was not unique to generic products because some manufacturers of branded medicines also sold their products directly to community pharmacies under price-volume agreements. This is one of the many economic issues that community pharmacists have to deal with in the efficient running of their businesses. Recent PBS reforms have created different remuneration schedules for generic and branded medicines resulting in these cost savings now being retained within the PBS.

\section{Can the bioavailability of bioequivalent products differ by up to $\mathbf{4 0} \%$ ?}

No, for two drugs to be bioequivalent, the $90 \%$ confidence intervals $(90 \% \mathrm{Cl})$ for the ratio of each pharmacokinetic parameter, $\mathrm{C}_{\max }$ and $\mathrm{AUC}$, must lie within the range $0.8-1.25$ (sometimes also expressed as $80-125 \%$ ).

The $90 \% \mathrm{Cl}$ of $0.8-1.25$ is a numerical index and not a direct measure of the difference in systemic concentrations of the active ingredient resulting from administration of the two products. It does not mean that the $\mathrm{C}_{\max }$ and $\mathrm{AUC}$ ratios estimated for each formulation can vary by -20 to $+25 \%$. In reality, for a product to fit within these relatively tight confidence limits the mean $A U C$ and $C_{\max }$ must be very close, and any difference in bioavailability is certainly less than $10 \% .{ }^{4}$

\section{Conclusion}

The bioequivalence criteria used in Australia have been defined and refined over many years and are internationally recognised as the acceptable criteria for assessing bioequivalence. ${ }^{1}$ There is persuasive evidence that the current internationally accepted limits and approaches to bioequivalence can accommodate all medicines. ${ }^{6,7}$

Only drugs that are marked as bioequivalent should be substituted for each other. Likewise, drugs that are not bioequivalent should not be exchanged.
To avoid confusion, healthcare professionals should, where possible, reinforce the name of the active ingredient in the medicine, when prescribing, dispensing and administering medicines to patients.

The authors acknowledge the helpful comments of Dr Greg Pearce (Medical Advisor, Alphapharm) and Mr Kos Sclavos (National President, Pharmacy Guild of Australia).

\section{References}

1. Birkett DJ. Generics - equal or not? Aust Prescr 2003;26:85-7.

2. Hassali A, Stewart K, Kong D. Quality use of generic medicines [editorial]. Aust Prescr 2004;27:80-1.

3. National Prescribing Service. Generic medicines: same difference? NPS News 2006;44.

http://www.nps.org.au/site.php?content=/html/news. php\&news=/resources/NPS_News/news44 [cited 2007 Mar 6]

4. Pearce GA, McLachlan AJ, Ramzan I. Bioequivalence: how, why, and what does it really mean? J Pharm Pract Res 2004;34:195-200.

5. Department of Health and Ageing. Schedule of Pharmaceutical Benefits. http://www.pbs.gov.au [cited 2007 Mar 6]

6. Benet LZ. Relevance of pharmacokinetics in narrow therapeutic index drugs. Transplant Proc 1999;31:1642-4.

7. Christians U, First MR, Benet LZ. Recommendations for bioequivalence testing of cyclosporine generics revisited. Ther Drug Monit 2000;22:330-45.

8. Uchegbu IF, Florence AT. Adverse drug events related to dosage forms and delivery systems. Drug Saf 1996;14:39-67.

9. Therapeutic Goods Administration. Best practice guideline on prescription medicine labelling. 2005. http://www.tga.gov. au/pmeds/pmbestpractice.htm [cited 2007 Mar 6]

10. Department of Health and Ageing. Guiding principles for medication management in the community. 2006. http://www.health.gov.au/internet/wcms/publishing.nsf/ content/apac-publications-guiding [cited 2007 Mar 6]

Each author has acted as a paid consultant to the pharmaceutical industry (including companies that manufacture innovator branded and generic medicines). Professors Ramzan and McLachlan are also members of the Pharmaceutical Subcommittee, Australian Drug Evaluation Committee.

\section{Dental notes}

\section{Prepared by Dr M McCullough of the Australian Dental Association}

\section{Frequently asked questions about generic medicines}

Habits formed in the early years after graduation often remain with us during our working life. Despite continuing professional development, when pressed for time or perhaps in a difficult clinical situation, we often revert to practices established early in our professional career. Prescribing drugs by brand name may be done out of habit, but this may not be in the best financial interest of our patients. We need to continually assess our prescribing habits and consider cost in our choice of drugs. There is usually no reason to be concerned about substituting a bioequivalent generic product for a branded product. To avoid confusion, always tell the patient the active ingredient of the medicine prescribed. When we write a prescription, we are recommending that our patients use a drug, not necessarily a brand. 